\title{
Circularly polarized vacuum field in three-dimensional chiral photonic crystals probed by quantum dot emission
}

\author{
S. Takahashi ${ }^{1,2}$ * Y. Ota ${ }^{1}$, T. Tajiri ${ }^{3}$, J. Tatebayashi ${ }^{1}$, S. Iwamoto ${ }^{1,3}$, and Y. Arakawa ${ }^{1,3}$ \\ ${ }^{1}$ Institute for Nano Quantum Information Electronics, University of Tokyo, \\ 4-6-1 Komaba, Meguro-ku, Tokyo 153-8505, Japan \\ ${ }^{2}$ Kyoto Institute of Technology, Matsugasaki, Sakyo-ku, Kyoto 606-8585, Japan \\ ${ }^{3}$ Institute of Industrial Science, University of Tokyo, \\ 4-6-1 Komaba, Meguro-ku, Tokyo 153-8505, Japan
}

(Dated: September 18, 2018)

\begin{abstract}
The quantum nature of light-matter interactions in a circularly polarized vacuum field was probed by spontaneous emission from quantum dots in three-dimensional chiral photonic crystals. Due to the circularly polarized eigenmodes along the helical axis in the GaAs-based mirror-asymmetric structures we studied, we observed highly circularly polarized emission from the quantum dots. Both spectroscopic and time-resolved measurements confirmed that the obtained circularly polarized light was influenced by a large difference in the photonic density of states between the orthogonal components of the circular polarization in the vacuum field.
\end{abstract}

PACS numbers: 42.70.Qs, 81.05.Xj, 77.22.Ej

Circular polarization $(\mathrm{CP})$ of light has attracted much attention for applications such as three-dimensional (3D) displays, bio-chemical sensing for chiral molecules, spintronics in solid states, and quantum information technology. One of the conventional ways of obtaining circularly polarized light, using a linear polarizer and a quarter-wave plate with a linearly polarized light source, induces some loss of energy, which prevents the use of this approach for achieving dense integration of optical circuits. Therefore, circularly polarized spontaneous emitters on the micrometer-scale have been studied by controlling spin states in solids with external magnetic fields [1, 2], by using the valley degree of freedom in monolayer chalcogenides [3], by using resonant multipolar moments in plasmonic nanoantennas [4], by modifying the vacuum field with periodic nano-structures [5, 6], and by selecting the local density of state (DOS) of the vacuum field in nano-structures [7 11]. In particular, control of the vacuum field enables us to tune spontaneous emission, with respect not only to its polarization but also the emission rates and directions [12]. However, these studied nanostructures for controlling the vacuum are 1D or (pseudo)2D structures. Since the electric/magnetic field in circularly polarized light has 3D helical symmetry, CP can be eigenpolarization over a broad spatial/spectral range only when the geometric structure has 3D helical symmetry [13]. Thus, additional spatial dimensions in 3D heli$\mathrm{cal} /$ chiral nano-structures will allow sophisticated control of circularly polarized light as well as deep insights into the circularly polarized vacuum field.

In this work, we successfully modified each circularly polarized component in the vacuum field independently by using semiconductor-based 3D chiral photonic crystals $(\mathrm{PhCs})$ [14, 15], and we demonstrated CP emission from quantum dots (QDs) embedded in the structures. Both the degree of circular polarization (DOP) of the emitted light and the radiative lifetime for each $\mathrm{CP}$ emission were measured to confirm the modulation of $\mathrm{CP}$ components in the vacuum field. Based on the circularly polarized band structure formed by the $3 \mathrm{D}$ chiral $\mathrm{PhCs}$, the former measurement showed a DOP as high as $\sim 50 \%$ for each $\mathrm{CP}$ at different wavelengths. The latter measurement at a particular wavelength showed $\mathrm{a} \sim 10 \%$ difference in the radiative lifetime between the orthogonal components of the $\mathrm{CP}$ emission. The wavelength dependencies in these two measurements were consistent each other. Although similar results have been reported in liquid crystals [16, 17], one of the important pieces of evidence presented for different emission rates between the orthogonal components of the $\mathrm{CP}$ has not been investigated in these reports. In addition, semiconductor-based $\mathrm{PhCs}$ can be monolithically compatible with current electrical circuits and future optical circuits, and can be developed into active devices driven by electrical current. Furthermore, manipulation of electron/hole spins in semiconductorbased nanostructures can be transferred to circularly polarized light through spin-photon interfaces [18, 19]. Our results obtained in the absence of a magnetic field will pave the way to realize circularly polarized micro-lasers without any spin injection in semiconductor systems, or optical readout system that can read superposed spin states by measuring the emission rates of the CPs.

One of the studied 3D chiral PhCs composed of a rotationally stacked woodpile structure [14, 15] is shown in Fig. 1(a). The structure is a stack of patterned thin plates of $225 \mathrm{~nm}$ thickness. These plates made of GaAs were fabricated by electron-beam lithography and dry and wet etching. The pattern in the plates was an array of rods with $120 \mathrm{~nm}$ width and $500 \mathrm{~nm}$ period. These 16 plates were stacked using a micro-manipulation technique [20 22] one-by-one with a $60^{\circ}$ in-plane rotation of each plate in order that three plates construct a single 


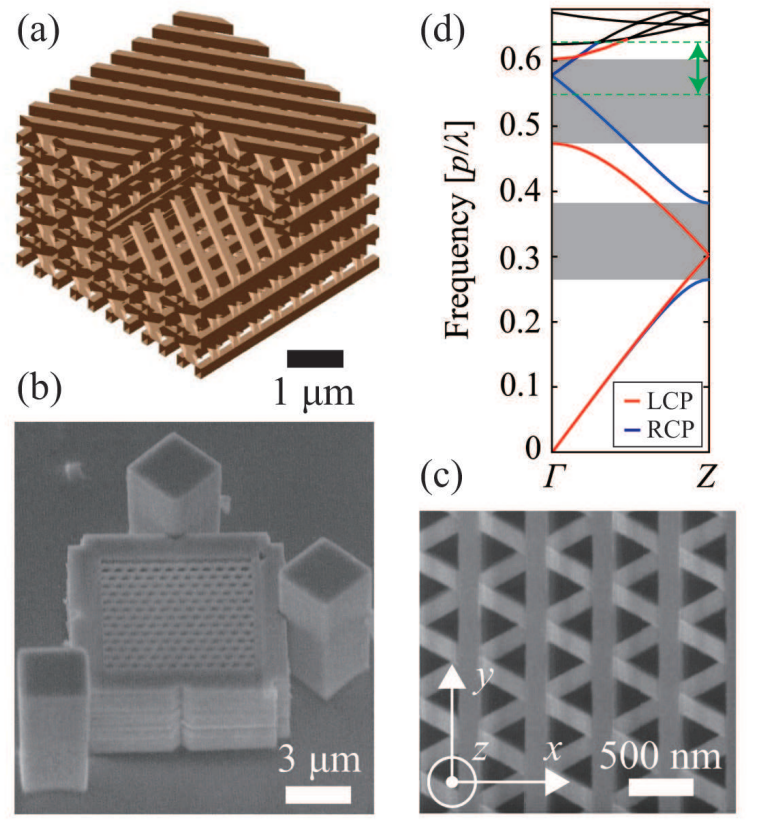

FIG. 1: (a) Schematic diagram of a rotationally-stacked woodpile structure. The top plate is removed and a part of the structure is cut away for clarity. (b) Scanning electron micrograph (SEM) image of the fabricated 3D PhC. Three posts are used as a guide for the micro-manipulation technique. (c) SEM image of the periodic rods. The crossing points arranged in a triangular lattice are aligned along the helical axis. (d) Numerically calculated photonic band structure along the helical axis for the chiral $\mathrm{PhC}$. The photonic band strongly polarized in LCP (RCP) is colored in red (blue). The polarization bandgaps for the opposite sense of $\mathrm{CP}$ appear in the two shaded regions. The frequency region indicated by the green double-headed arrow is measured in this study.

helical unit as a space group with $3_{1}$ screw operation. Hence, the pitch of the helix was $p=675 \mathrm{~nm}$. In this study, we prepared two kinds of plates: active (passive) plates with (without) three layers of InAs self-assembled QDs having a density of $10^{10} / \mathrm{cm}^{2}$. The studied chiral structures contained QDs in the middle three plates. One of the fabricated chiral PhCs is shown in Fig. 1(b) and (c). The $x(y)$ axis is defined to be orthogonal (parallel) to the rods in the top plate, and the $z$ axis is parallel to the helical axis. For comparison, we also fabricated an achiral structure by stacking the plates without any in-plane rotation, meaning that the rods were aligned throughout the stacking direction. Note that the chirality of similar structures was previously confirmed by measuring optical activity, namely, optical rotation 14 and circular dichroism [15]. Also, the QDs were naturally $p$-doped due to carbon incorporation during their growth by metal-organic chemical vaper deposition.

The periodically stacked rods formed a photonic band structure along the helical axis, as confirmed in numerical simulations by a plane wave expansion method shown

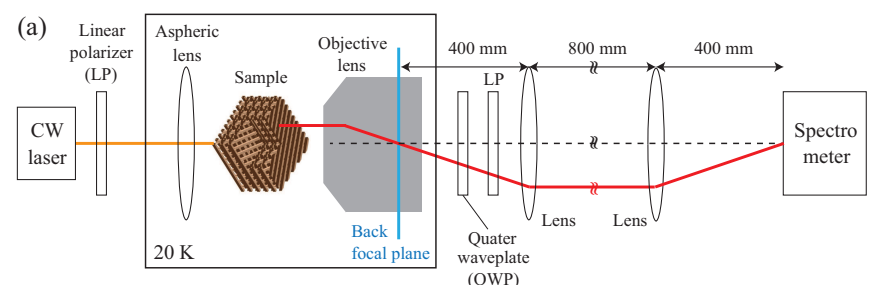

(b)

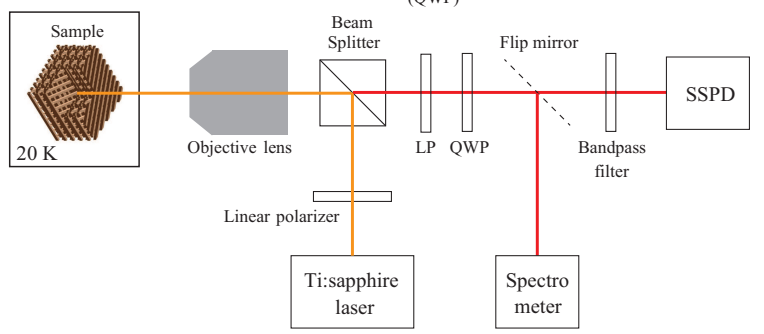

FIG. 2: Schematics diagrams of the experimental setups for the DOP measurement (a) and the radiation lifetime measurement (b). In (a), the back focal plane is transferred by the 4 -f system, and its center part is selected by choosing a single photodiode chip out of the $2 \mathrm{D}$ array. In (b), after checking the emission spectra with the spectrometer, the PL signal at a particular wavelength is selected by a bandpass filter, and its decay curve is measured by the SSPD.

in Fig. 1(d). Here, $\Gamma$ and $Z$ respectively represent the origin and edge of the first Brillouin zone, corresponding to the $z$ direction in the real space. Since the optical modes in the red/blue colored bands in Fig. 1(d) were strongly polarized in left/right-handed circular polarization (LCP/RCP), the chiral PhC inherently had two polarization bandgaps in different wavelength regions shaded in Fig. 1(d) [15, 23]. In what follows, we focus on one of the polarization bandgaps in the region of 0.48 $<p / \lambda<0.60(1122 \mathrm{~nm}<\lambda<1421 \mathrm{~nm})$ and its band edge at $p / \lambda=0.60(\lambda=1122 \mathrm{~nm})$, which is covered by the emission spectrum of the ensemble QDs 24].

First, we performed photoluminescence (PL) measurements by optically exciting InAs wetting layers near the QDs in the PhCs and by detecting the light emitted from the QDs. The measurement setup is shown in Fig. 2(a). The excitation laser light had a power of $40 \mu \mathrm{W}$ and the linearly polarized output light was focused on the samples into a $3 \mu \mathrm{m}$ spot size by a $60 \times$ aspheric lens having a numerical aperture of 0.54 . The wavelength of the laser light was $980 \mathrm{~nm}$, at which GaAs is transparent. Then, the broadband infrared luminescence from the backside of the samples was collimated by a $50 \times$ objective lens with a numerical aperture of 0.55 . The collimated luminescence was passed through a quarter-wave plate and a polarizer and was detected by a spectrometer equipped with a 2D array of InGaAs photodiodes. The samples, as well as the aspheric lens and the objective lens, were cooled down to $20 \mathrm{~K}$, and no magnetic field was applied. Note that since the circularly polarized band structure was created only in the direction along the helical axis, 

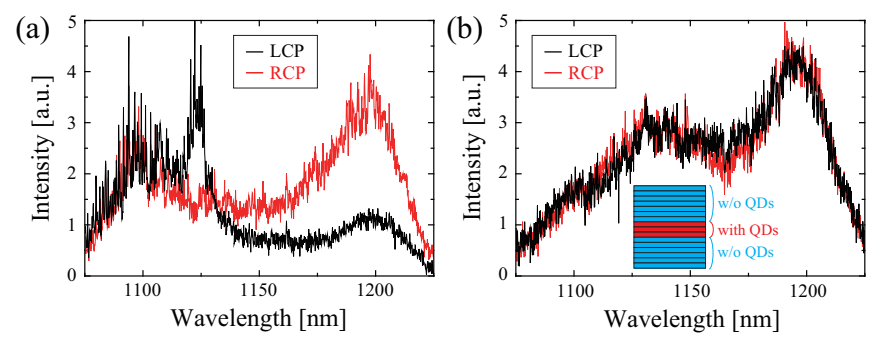

(c)

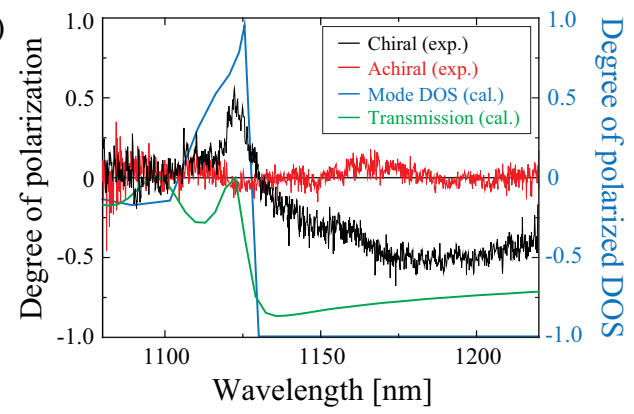

FIG. 3: (a) PL spectra for LCP (RCP) component in black (red) for the chiral $\mathrm{PhC}$ containing QDs in the middle three plates. Clear intensity differences appear at around $1120 \mathrm{~nm}$ and $1200 \mathrm{~nm}$ in the opposite rotation senses. (b) PL spectra for LCP (RCP) component in black (red) for the achiral structure. No intensity difference appears throughout the QD emission wavelength. Inset shows a schematic diagram of the layered achiral/chiral $\mathrm{PhC}$ with QDs in the middle three plates. (c) DOP as a function of wavelength obtained from (a) in black, from (b) in red, from the $\rho_{L C P / R C P}(\omega)$ in blue, and from the calculated transmittance in green. At the polarization band edge at around $1120 \mathrm{~nm}$, the QD emission from the chiral structure and the $\rho_{L C P / R C P}$ showed positive DOP values, while the transmission showed zero DOP.

we selectively detected the emitted light possessing momentum parallel to the helical axis using a 4-f system. Two additional lenses with a focal length of $400 \mathrm{~mm}$ and a numerical aperture of 0.03 were focused on the back focal plane of the objective lens and on the slit of the spectrometer, respectively. The center region of the back focal plane transferred by this 4 -f system was selectively detected by choosing a single InGaAs photodiode of size $20 \mu \mathrm{m} \times 20 \mu \mathrm{m}$ out of the $640 \times 5122 \mathrm{D}$ array.

In order to observe the direct effect of the modulated circularly polarized vacuum field on the QD emission, we also performed time-resolved PL measurements. The setup is schematically shown in Fig. 2(b). The QDs in the PhCs were excited by a Ti:sapphire mode-locked laser with a central wavelength of $890 \mathrm{~nm}$, a pulse duration time of $1 \mathrm{ps}$, a repetition rate of $80 \mathrm{MHz}$, and a time-averaged power of $1.5 \mu \mathrm{W}$. The pulsed laser light was linearly polarized and focused in a $3 \mu$ m-diameter spot on the sample by a $50 \times$ objective lens with a numerical aperture of 0.65 . After selecting the wavelength and polarization of the emitted light, a superconductive single photon detector (SSPD) was used for measuring the PL intensity decay curve with a timing resolution of $\sim 25$ ps.

Figure 3(a) shows the PL intensity of LCP (RCP) in black (red) as a function of wavelength for the chiral $\mathrm{PhC}$ containing QDs in the middle three plates. The LCP intensity was stronger than the RCP intensity in a narrow wavelength region at around $1120 \mathrm{~nm}$, whereas the LCP intensity was weaker than the RCP intensity in a broad wavelength region at around $1200 \mathrm{~nm}$. Such a clear difference of PL intensity between LCP and RCP did not appear for the achiral structure, as shown in Fig. 3(b). Note that the two broad peaks at around $1100 \mathrm{~nm}$ and $1200 \mathrm{~nm}$ were the ensemble QD emission from the first excited state and the ground state, respectively 24].

From these results, we calculated the DOP from the detected intensity of LCP and RCP using $D O P=$ $\left(I_{L C P}-I_{R C P}\right) /\left(I_{L C P}+I_{R C P}\right)$. Figure $3(\mathrm{c})$ plots the $\mathrm{DOP}$ as a function of the emitted wavelength for the chiral (black) and the achiral (red) structures. This figure clearly shows that the QDs in the chiral PhC emitted narrowband LCP light in the short wavelength region, whereas broadband RCP light was obtained in the long wavelength region. The absolute value of the obtained DOP was as high as $\sim 50 \%$, which is sufficiently larger than that for the achiral structure. Here, we define "mode DOS" $\rho_{L C P / R C P}(\omega)$ of the vacuum field for the particular LCP/RCP electromagnetic mode in Fig. $1(\mathrm{~d})$ at an angular frequency $\omega$. Note that $\rho_{L C P / R C P}(\omega)$ is only a part of the total DOS, $\rho_{\text {total }}(\omega)$, which also includes the DOS of the other modes propagating in the other 3D directions. In the polarization band gap formed by the chiral $\mathrm{PhC}, \rho_{L C P}(\omega)$ was suppressed. Therefore, the QDs prefer to emit RCP rather than LCP light in the long wavelength region. On the other hand, in the short wavelength region at around the polarization band edge, $\rho_{L C P}(\omega)$ was greatly enhanced, and LCP light was strongly emitted from the QDs. In fact, the degree of polarized DOS $\left(\rho_{L C P}-\rho_{R C P}\right) /\left(\rho_{L C P}+\rho_{R C P}\right)$ derived from the band structure in Fig. $1(\mathrm{~d})$ showed positive values at around the band edge, as depicted by the blue curve in Fig. 3(c).

Here, we consider the effect of circular dichroism, which works as a filter for $\mathrm{CP}$ in the transmission. We performed numerical simulations based on the finitedifference time-domain (FDTD) method for the chiral $\mathrm{PhC}$ with periodic boundary conditions in the in-plane $(x$ and $y$ ) directions and with perfectly matched layers attached at $10 \mu \mathrm{m}$ away from the center of the structure in the stacking $(z)$ direction. The $\mathrm{PhC}$ was normally irradiated with a pulsed plane wave having each CP. The pulse duration was a single cycle for the central wavelength of $1500 \mathrm{~nm}$. The recorded time-dependent electromagnetic field of the transmitted light for each CP incidence was analyzed in frequency space using a Fourier transform. From the obtained transmittance spectrum for each incident $\mathrm{CP}$, the DOP spectrum was plotted (green curve in Fig. 3(c)). While the QD emission in the chiral $\mathrm{PhC}$ 

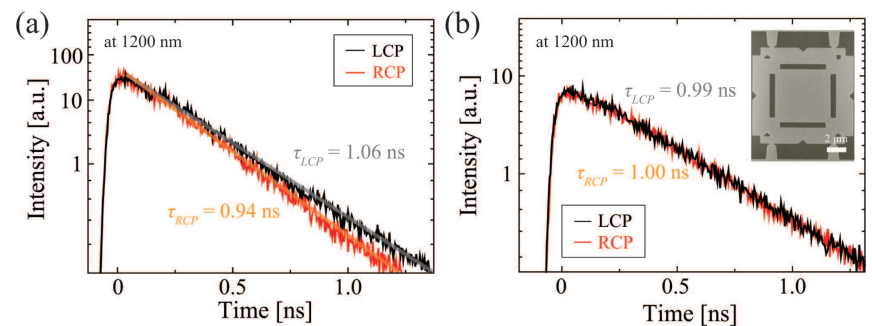

(c)

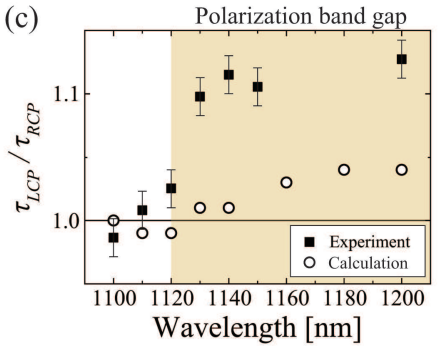

FIG. 4: (a) Time-decay of the PL intensity for the two orthogonal components of the $\mathrm{CP}$ at $1200 \mathrm{~nm}$ wavelength for the chiral $\mathrm{PhC}$. The PL intensity is normalized for clarity. The gray and orange lines are single exponential fitted curves for the LCP and RCP decay curves, respectively. The suppression of the $\rho_{L C P}$ caused an imbalance between LCP and RCP components in $\rho_{\text {total }}$, resulting in the longer radiative lifetime for LCP than that for RCP. (b) Time-decay of the PL intensity at $1200 \mathrm{~nm}$ wavelength for a single active plate containing QDs, as shown in the inset. No difference was observed between the two CPs. (c) Experimentally obtained radiative lifetime ratio $\tau_{L C P} / \tau_{R C P}$ as a function of wavelength for the chiral $\mathrm{PhC}$ (solid squares). Numerically obtained lifetime ratio is also plotted as open circles, showing qualitative agreement with the experimental results. A lifetime difference between the CPs appeared in the long wavelength region, which is consistent with the polarization bandgap.

showed a positive DOP at the band edge at around 1120 $\mathrm{nm}$, the transmission showed zero DOP. Even if $\rho_{L C P}$ is enough large, the LCP transmittance reaches unity at most, the same as the RCP transmittance. Therefore, the sign difference of the measured DOP indicates that the measured $\mathrm{CP}$ emission at around $1120 \mathrm{~nm}$ resulted from the large $\rho_{L C P}$ at the polarization band edge, rather than the circular dichroism effect.

We also measured other chiral $\mathrm{PhCs}$ with different structural parameters, such as different rod widths from $120 \mathrm{~nm}$ to $150 \mathrm{~nm}$ and $190 \mathrm{~nm}$, and different plate thicknesses from $225 \mathrm{~nm}$ to $240 \mathrm{~nm}$. Since the vacuum field is modified by the CP band structure, tuning the structural parameters of the chiral $\mathrm{PhC}$ modulated the band structure, resulting in a shift of the wavelength for the DOP peak/dip in Fig. 3(c). In fact, the obtained DOP spectra for the chiral PhCs with different structural parameters showed red shifts of the wavelength for the DOP peak and dip [24]. These results confirmed that the DOP spectrum in Fig. 3(c) was significantly influenced by the modified circularly polarized vacuum field in the chiral $\mathrm{PhC}$.
Figure 4(a) shows the time-decay of the PL intensity obtained by the time-resolved PL measurements for the chiral $\mathrm{PhC}$ at a wavelength of $1200 \mathrm{~nm}$ in the polarization bandgap. By fitting with a single exponential function at around the beginning of the intensity decay, the radiative lifetime of LCP, $\tau_{L C P}=1.06 \mathrm{~ns}$ was longer than that of RCP, $\tau_{R C P}=0.94 \mathrm{~ns}$. Since radiative lifetime is proportionally influenced by the total DOS $\rho_{\text {total }}$ [12, 25], the lifetime difference indicates that $\rho_{L C P}$ is smaller than $\rho_{R C P}$ in the polarization bandgap. Note that there was no difference between $\tau_{L C P}$ and $\tau_{R C P}$ for an active plate without the rod pattern, as shown in Fig. 4(b), confirming that the lifetime difference of the CPs in Fig. 4(a) originates from the chiral structure. Figure 4(c) plots the radiative lifetime ratio $\tau_{L C P} / \tau_{R C P}$ for various wavelength in the chiral PhC. A lifetime difference between LCP and RCP appeared in the wavelength region longer than $1120 \mathrm{~nm}$, which is consistent with the polarization band edge obtained in Fig. 3(c).

We performed numerical calculations of the lifetime ratio 24]. Using the FDTD method, the Poynting vectors were integrated over a surface enclosing the circularly polarized light source in the chiral $\mathrm{PhC}[26,27]$. The numerical results plotted in Fig. $4(\mathrm{c})$ show that $\tau_{L C P} / \tau_{R C P}$ $>1$ in the long wavelength region, which is qualitatively consistent with the experimental results. The quantitative difference between the experimental and numerical results was partly caused by the small in-plane size of the numerical chiral $\mathrm{PhC}$, owing to computational time restrictions 24].

In spite of the high DOP $\sim 50 \%$ in Fig. 3(c), the obtained lifetime difference between the orthogonal components of the CP was only $\sim 10 \%$ in both the experiment and the calculation. This is because the DOP is influenced only by $\rho_{L C P / R C P}(\omega)$ for particular modes propagating in the $z$ direction due to the selection of momentum using a single photodiode. On the other hand, the lifetime is derived from the total DOS $\rho_{\text {total }}$ which is the integral for all modes propagating in any $3 \mathrm{D}$ direction and having any polarization. Hence, the large difference between $\rho_{L C P}$ and $\rho_{R C P}$ was almost averaged by the DOS for all other modes. The subsequent selection of momentum by choosing a single photodiode out of the 2D array did not affect the lifetime.

Another possible reason for the small lifetime difference compared with the DOP is the effect of spin relaxation in the QDs, and this should be taken into account. Rate equations for the population of electron-hole pairs emitting LCP (RCP), $P_{L C P}\left(P_{R C P}\right)$, including spin relaxation in the absence of a magnetic field, are written as [28],

$$
\begin{aligned}
& \frac{d P_{L C P}}{d t}=-\frac{P_{L C P}}{\tau_{L C P}}-\frac{P_{L C P}-P_{R C P}}{\tau_{\text {spin }}}, \\
& \frac{d P_{R C P}}{d t}=-\frac{P_{R C P}}{\tau_{R C P}}+\frac{P_{L C P}-P_{R C P}}{\tau_{\text {spin }}} .
\end{aligned}
$$


Here, $\tau_{\text {spin }}$ is the electron/hole spin relaxation time $T_{1}$. For both electrons and holes in ensemble self-assembled QDs, $T_{1}>T_{2}>T_{2}^{*}=1-10 \mathrm{~ns}[29]$ for spin decoherence (dephasing) time $T_{2}\left(T_{2}^{*}\right)$. This time scale is comparable to the obtained radiative lifetime for both CPs. In these conditions, as well as $P_{L C P}=P_{R C P}$ at $t=0$ because of the linearly polarized excitation pulse, the rate equations showed a significant difference in time-decay between $P_{L C P}$ and $P_{R C P}$ even in the presence of the spin relaxation. Note that under the condition that the spin relaxation time is much shorter than $\tau_{L C P(R C P)}$, such as electron-hole exchange interactions in non-doped QDs, the time-decays of $P_{L C P}$ and $P_{R C P}$ coincide. This was not the case in this study where positively-charged excitons $X^{+}$were mainly involved due to the natural $p$ doping in the QDs. Therefore, we can conclude that the spin relaxation in the QDs has little effect on the measured lifetime difference. The lifetime difference could be enhanced by band engineering in the in-plane directions, or by employing different structures that have helical axes in various directions, such as the srs net [30].

At the polarization band edge where $\rho_{L C P}$ was enhanced, both the time-resolved PL measurement and the calculation showed $\tau_{L C P} / \tau_{R C P} \sim 1$ in Fig. 4(c), whereas $\tau_{L C P} / \tau_{R C P}>1$ in the polarization bandgap where $\rho_{L C P}$ was suppressed. This difference is due to the spatial distribution of the electromagnetic field. Another numerical calculation showed that the in-plane distribution of the LCP electric field at the band edge was relatively localized in the rods compared with that at the band gap [24]. Therefore, by considering the local DOS in the rods, $\tau_{L C P} / \tau_{R C P}$ at the band edge is sensitive to averaging for all QD positions in the rods and reaches unity, whereas $\tau_{L C P} / \tau_{R C P}>1$ is maintained in the band gap. A similar averaging effect for the lifetime or DOS at band edges has been experimentally observed and discussed in 2D PhCs 31, 32]. However, if a single QD could be positioned accurately in the chiral $\mathrm{PhC}$, the band edge effect would potentially show a large reduction of $\tau_{L C P}$. In fact, $\tau_{L C P} / \tau_{R C P}$ was shown to be as low as 0.83 at the band edge for a particular position of a light source [24].

In conclusion, we investigated spontaneous emission from QDs embedded in 3D chiral PhCs, and confirmed the modulation of the $\mathrm{CP}$ components in the vacuum field. The QDs in the chiral structure emitted highly circularly polarized light with a DOP $\sim 50 \%$ in each sense of CP (right- and left-handed) at the polarization band edge and in the polarization bandgap, respectively. From the results of spectroscopic and time-resolved measurements, the obtained circularly polarized light was revealed to be directly influenced by the modified DOS of the vacuum field. These results could be a large step towards the realization of circularly polarized light emitting diodes, lasers, and sensors in semiconductor systems.

This work was supported by Grant-in-Aid for Scientific Research (16H06085, 26889018), Grant-in-Aid for Specially Promoted Research (15H05700), and Grant-in-Aid for Scientific Research on Innovative Areas (15H05868).

* Electronic address: shuntaka@kit.ac.jp

[1] H. Ando, T. Sogawa, and H. Gotoh, Appl. Phys. Lett. 73, 566 (1998).

[2] S. Iba, et al., Appl. Phys. Lett. 98, 081113 (2011).

[3] Y. J. Zhang, et al., Science 344, 725 (2014).

[4] S. S. Kruk, et al., ACS Photonics 1, 1218 (2014).

[5] K. Konishi, et al., Phys. Rev. Lett. 106, 057402 (2011).

[6] S. V. Lobanov, et al., Phys. Rev. B 92, 205309 (2015).

[7] I. J. Luxmoore, et al., Phys. Rev. Lett. 110, 037402 (2013).

[8] J. Petersen, J. Volz, and A. Rauschenbeutel, Science 346, 67 (2014).

[9] I. Söllner, et al., Nat. Nanotech. 10, 775 (2015).

[10] A. B. Young, et al., Phys. Rev. Lett. 115, 153901 (2015).

[11] R. J. Coles, et al., Nat. Commun. 7, 11183 (2016).

[12] M. O. Scully and M. S. Zubairy, Quantum Optics (Cambridge University Press, Cambridge, 1997).

[13] J. K. Gansel, et al., Science 325, 1513 (2009).

[14] S. Takahashi, et al., Opt. Express 21, 29905 (2013).

[15] S. Takahashi, et al., Appl. Phys. Lett. 105, 051107 (2014).

[16] K. L. Woon, et al., Phys. Rev. E 71, 041706 (2005).

[17] H. Coles and S. Morris, Nat. Photon. 4, 676 (2010).

[18] K. D. Greve, et al., Nature 491, 421 (2012).

[19] W. B. Gao, et al., Nature 491, 426 (2012).

[20] K. Aoki, et al., Nat. Mater. 2, 117 (2003).

[21] K. Aoki, et al., Nat. Photon. 2, 11 (2008).

[22] A. Tandaechanurat, et al., Nat. Photon. 5, 91 (2011).

[23] J. C. W. Lee and C. T. Chan, Opt. Express 13, 8083 (2005).

[24] See EPAPS Document No.XXXX for characterization details and additional data.

[25] L. Novotny and B. Hecht, Principles of Nano-Optics (Cambridge University Press, Cambridge, 2012).

[26] Y. Xu, et al., J. Opt. Soc. Am. B 16, 465 (1999).

[27] R. K. Lee, et al., J. Opt. Soc. Am. B 17, 1438 (2000).

[28] R. J. Seymour and R. R. Alfano, Appl. Phys. Lett. 37, 231 (1980).

[29] C. Kloeffel and D. Loss, Annu. Rev. Condens. Matter Phys. 4, 51 (2013).

[30] M. Saba, et al., Phys. Rev. Lett. 106, 103902 (2011).

[31] M. Fujita, et al., Science 308, 1296 (2005).

[32] Q. Wang, S. Stobbe, and P. Lodahl, Phys. Rev. Lett. 107, 167404 (2011). 